Sánchez-Alonzo, D.M., Solís-Marín, F.A., \& Conejeros-

Vargas, C.A. (2021). Nuevos registros de la familia

Cucumariidae (Holothuroidea: Dendrochirotida)

en el Pacífico mexicano. Revista de Biología

Tropical, 69(S1), 375-386. DOI 10.15517/rbt.

v69iSuppl.1.46369

DOI 10.15517/rbt.v69iSuppl.1.46369

\title{
Nuevos registros de la familia Cucumariidae (Holothuroidea: Dendrochirotida) en el Pacífico mexicano
}

\author{
Daniel M. Sánchez-Alonzo ${ }^{1 *}$ \\ Francisco A. Solís-Marín ${ }^{2}$ \\ Carlos A. Conejeros-Vargas ${ }^{3}$
}

1. Facultad de Ciencias, Universidad Nacional Autónoma de México. Circuito exterior s/n, C.P. 04510, Ciudad de México, México; alonzodanniel@gmail.com (*Correspondencia).

2. Colección Nacional de Equinodermos "Dra. Ma. Elena Caso Muñoz", Laboratorio de Sistemática y Ecología de Equinodermos, Instituto de Ciencias del Mar y Limnología, Universidad Nacional Autónoma de México, C.P. 04510, Ciudad de México, México; fasolis@cmarl.unam.mx

3. Posgrado en Ciencias del Mar y Limnología, Universidad Nacional Autónoma de México; Av. Ciudad Universitaria 3000, C.P. 04510, Ciudad de México, México; conejeros@ciencias.unam.mx

Recibido 14-VII-2020. Corregido 25-VIII-2020. Aceptado 10-XI-2020.

\begin{abstract}
New records of the family Cucumariidae (Holothuroidea: Dendrochirotida) for the Mexican Pacific

Introduction: Cucumarids are a cosmopolitan group of sea cucumbers that inhabit rocky-sandy sediments, from the intertidal zone to the hadal zone. Objective: To present new records for the Mexican Pacific. Methods: We applied stereoscope and scanning electron microscopy to external and internal morphology of specimens in the collection of the National Autonomous University, Mexico. Results: We present a taxonomic synopsis of cucumarids in the Mexican Pacific and review their taxonomy on the ossicles. The taxonomic identity of Pseudocnus curatus, Pseudocnus dubiosus and Pseudocnus lubricus is confirmed, and the presence of Trachythyone peruana is ruled out. Conclusions: Four new records are presented for the Mexican Pacific: Leptopentacta nina, Pseudocnus curatus, Pseudocnus dubiosus and Pseudocnus lubricus.
\end{abstract}

Key words: sea cucumbers; Mexican Pacific; biodiversity; taxonomy; morphology.

El grupo de los cucumáridos está conformado por pepinos de mar dendroquirótidos que se caracterizan por poseer 10 tentáculos y el cuerpo más o menos cilíndrico o fusiforme, a veces muy curvado, sin suela ventral claramente definida y con las aberturas oral y anal en posiciones terminal o dorsal. Los pies ambulacrales se suelen restringir a los radios o ambulacros, aunque pueden estar dispersos y extendidos sobre los interradios y también en la superficie dorsal, la cual nunca está ornamentada por papilas. Presenta un anillo calcáreo sin prolongaciones posteriores o con prolongaciones cortas no divididas, y ámpulas tentaculares rudimentarias. Las espículas de la pared del cuerpo se caracterizan por tener formas de botones, placas perforadas y canastas (estas últimas exclusivamente en la subfamilia 
Colochirinae) (Caso, 1961; Pawson, 1982; Borrero-Pérez, Benavides-Serrato, \& DíazSánchez, 2012; Smirnov, 2012).

Los cucumáridos son un grupo cosmopolita que habita en sedimentos rocoso-arenosos, desde la zona intermareal hasta la zona hadal. El primer registro de un cucumárido para el Pacífico mexicano fue realizado por Semper (1868) durante la descripción de Cucumaria californica Semper, 1868 con ejemplares procedentes de la costa de Mazatlán, Sinaloa. Poco más de 70 años después, Deichmann (1941) reportó tres especies de cucumáridos para el Pacífico mexicano: Leptopentacta nova Deichmann, 1941 para la bahía de Santa María, Baja California Sur, Leptopentacta panamica Deichmann, 1941 para la bahía de Tangolunda, Oaxaca, y Thyonella mexicana (Deichmann, 1941) para la bahía de San Gabriel, isla Espíritu Santo, Baja California.

En su tesis doctoral, Caso (1961) reportó tres especies pertenecientes a la familia Cucumariidae para México, dos para el Pacífico $(C$. californica y Trachythyone peruana (Semper, 1868)). Posteriormente, Caso (1984) describió a Parathyonacta bonifaznunoi Caso, 1984 y erigió al género monoespecífico Parathyonacta basándose en la morfología interna y externa, así como la forma y combinación de las espículas y comparándola con Thyonella mexicana descrita por Deichmann (1941). En el trabajo de Solís-Marín, Arriaga-Ochoa, LaguardaFigueras, Frontana-Uribe, y Durán-González (2009) los autores sinonimizaron formalmente a $P$. bonifaznunoi con $T$. mexicana. Panning (1962) describió a Pseudocnus californicus (Semper, 1868) con material procedente de la bahía de los Ángeles, Baja California dentro del área correspondiente al golfo de California. Bergen (1996) reportó tres especies de cucumáridos para la costa occidental de Baja California, México, particularmente, denota la existencia de Cucumaria piperata (Stimpson, 1864) en isla San Benito, Baja California. Solís-Marín y Laguarda-Figueras (1999) describieron a Cucumaria flamma, a partir de organismos procedentes de playa Pedregosa en Zihuatanejo, Guerrero.

Una de las recopilaciones más amplias de los registros de equinodermos para el litoral del Pacífico mexicano fue el trabajo de HoneyEscandón, Solís-Marín, y Laguarda-Figueras (2008) en el que reportan siete especies de la familia Cucumariidae: Cucumaria crax Deichmann, 1941, C. flamma, L. nova, P. californicus, T. peruana, Neocucumis veleronis (Deichmann, 1941) y Abyssocucumis abyssorum (Théel, 1886); estas últimas dos especies fueron transferidas a la familia Thyonidiidae Heding \& Panning, 1954 por Smirnov (2012). Solís-Marín et al. (2009) reportaron a C. crax, C. flamma, P. californicus, T. mexicana y $T$. peruana para la región del golfo de California.

Ocho años más tarde, Solís-Marín, Caballero-Ochoa, Frontana-Uribe, Laguarda-Figueras, y Durán-González (2017) reportaron para el litoral mexicano un total de 13 especies de pepinos de mar de la familia Cucumariidae, ocho de ellas para el Pacífico. En el trabajo más reciente en el que se incluye un listado de equinodermos para la costa del estado de Michoacán, Nava et al. (2019) reportaron cinco especies de cucumáridos.

A pesar de que existen diversos trabajos en donde se registran listados de especies de holoturoideos, el esfuerzo de muestreo dirigido exclusivamente hacia la familia Cucumariidae no ha sido suficiente y por consiguiente los estudios taxonómicos existentes de esta familia son insuficientes. La justificación de este trabajo se centra en generar un mayor conocimiento del grupo para el Pacífico mexicano, dando a conocer nuevos registros de especies pertenecientes a la familia Cucumariidae que fueron localizados durante el análisis de los reportes previos y del material depositado en la Colección Nacional de Equinodermos del Instituto de Ciencias del Mar y Limnología de la Universidad Nacional Autónoma de México. 


\section{MATERIALES Y MÉTODOS}

Los especímenes depositados en la Colección Nacional de Equinodermos "Dra. María Elena Caso Muñoz", del Instituto de Ciencias del Mar y Limnología, Universidad Nacional Autónoma de México (ICML-UNAM), preservados en etanol al $70 \%$ fueron taxonómicamente revisados. Para la corroboración de los ejemplares a nivel de especie, se compararon los caracteres morfológicos externos e internos con ayuda de un microscopio estereoscópico Olympus SZX7. Como caracter taxonómico principal se consideró la forma y combinación de las espículas de diversas regiones del cuerpo. Para observar las espículas al microscopio óptico Nikon ECLIPSE 55i, se realizó un corte de tejido (aprox. de $5 \mathrm{~mm}^{2}$ ) de la pared del cuerpo de la superficie dorsal y ventral, así como de algunas regiones como los pies ambulacrales, el introverso, los tentáculos y de la región anal. La extracción de la muestra se realizó con unas tijeras o bisturí, la manipulación del tejido se llevó a cabo con una pinza de punta fina; el tejido se colocó sobre un portaobjetos, y se le añadió una o dos gotas de hipoclorito de sodio (cloro comercial) en el que se diluyó el tejido. Cuando las espículas se precipitaron sobre el portaobjetos se procedió a observar la preparación en el microscopio óptico (10X y 20X). Para la toma de fotografías de microscopía electrónica de barrido (MEB) se siguió la metodología propuesta por Solís-Marín et al. (2009). Para observar la muestra y tomar fotografías de MEB se utilizó un microscopio S-2460N Hitachi en el Laboratorio Nacional de la Biodiversidad I del Instituto de Biología (IB), UNAM. Una vez obtenidas las fotografías de MEB, se editaron usando el programa Adobe Photoshop CS6 portable.

A partir de la revisión de literatura y de los especímenes depositados en el ICML-UNAM se generó el listado taxonómico actualizado para la familia Cucumariidae en el Pacífico mexicano. La organización general de la sinopsis taxonómica está basada en los criterios de clasificación de Pawson \& Fell (1965), Smirnov (2012) y Miller et al. (2017).

Abreviaturas: CAS-IZ: California Academy of Science-Invertebrate Zoology, San Francisco, California, Estados Unidos. LACM: Los Angeles County Museum, Los Angeles, California, Estados Unidos. MCZ: Museum of Comparative Zoology, Boston, Massachusstes, Estados Unidos. USNM: National Museum of Natural History, Smithsonian Institution, Washington, D.C., Estados Unidos. ZMH: Zoologisches Museum Hamburg, Alemania.

\section{RESULTADOS}

Como resultado de la revisión de literatura se encontró un total de 12 especies de cucumáridos para el Pacífico mexicano (Tabla 1). Adicionalmente, en el presente trabajo se revisaron 68 especímenes depositados en la colección ICML-UNAM que corresponden a cuatro especies pertenecientes a la familia Cucumariidae, incluidas en dos subfamilias y cuatro géneros: Leptopentacta nina Deichmann, 1941, Pseudocnus curatus (Cowles, 1907), Pseudocnus dubiosus (Semper, 1868) у Pseudocnus lubricus (Clark, 1901); estas especies corresponden a nuevos registros para el Pacífico mexicano y son mencionadas a continuación. 
TABLA 1

Sinopsis taxonómica de las especies pertenecientes a la familia Cucumariidae reportadas para el Pacífico mexicano, incluyendo nuevos registros (N.R.)

TABLE 1

Taxonomic synopsis of species belonging to the Cucumariidae family reported for the Mexican Pacific, including new records (N.R.)

Clase Holothuroidea Selenka, 1867

Subclase Actinopoda Ludwig, 1891

Orden Dendrochirotida Grube, 1840

Suborden Cucumariina Smirnov, 2012

Familia Cucumariidae Ludwig, 1894

Subfamilia Colochirinae Panning, 1949

Género Leptopentacta Clark, 1938

Leptopentacta nina Deichmann, 1941 N.R.

Leptopentacta nova Deichmann, 1941

Leptopentacta panamica Deichmann, 1941

Género Thyonella Verrill, 1872

Thyonella mexicana (Deichmann, 1941)

Subfamilia Cucumariinae Ludwig, 1894

Género Cucumaria de Blainville, 1830

Cucumaria californica Semper, 1868

Cucumaria crax Deichmann, 1941

Cucumaria flamma Solís-Marín \& Laguarda-Figueras, 1999

Cucumaria piperata (Stimpson, 1864)

Género Pseudocnus Panning, 1949

Pseudocnus californicus (Semper, 1868)

Pseudocnus curatus (Cowles, 1907) N.R.

Pseudocnus dubiosus (Semper, 1868) N.R.

Pseudocnus lubricus (Clark, 1901) N.R.

\section{Sistemática}

Leptopentacta nina Deichmann, 1941

(Fig. 1A, 1B, 1C, 1D, Fig. 3)

Leptopentacta nina Deichmann, 1941: 97-98; Alvarado, Solís-Marín, \& Ahearn, 2010: 50 (lista); Alvarado et al., 2017: 281 (lista).

Diagnosis (modificada de Deichmann, 1941): Cuerpo con forma de trapecio alargado, con el extremo anterior cuadrado y el posterior afilado (Fig. 1A). Con 10 tentáculos. Piel rígida y áspera, con surcos en toda la pared del cuerpo. Zona anal con estructuras en forma de dientes anales. Anillo calcáreo simple con 10 piezas. Bivium y trivium bien diferenciados, con papilas (bivium) y ventosas (trivium). Árboles respiratorios presentes. Espículas de la pared del cuerpo tipo canasta y botones abollonados (Fig. 1B, Fig. 1C). En el introverso y tentáculos presentan barrotes perforados, curvados (Fig. 1D).

Referencia de identificación: Deichmann, 1941: 97-98, lám. 15, Figs. 1-12.

Material examinado: 26 especímenes. ICML-UNAM 2379, siete especímenes, a $93 \mathrm{~m}$ de punta Piaxtla, Sinaloa, México, $23^{\circ} 40$ '13.22" N \& 106 51 '27.58" W; ICMLUNAM 3320, nueve especímenes, a $37 \mathrm{~m}$ de punta Piaxtla, Sinaloa, México, 23 ${ }^{\circ} 40^{\prime} 53.67^{\prime}$ ' $\mathrm{N} \& 106^{\circ} 49^{\prime} 47.47^{\prime \prime} \mathrm{W}, 50 \mathrm{~m}$; ICML-UNAM 3640, dos especímenes, estero Tastiola, golfo de California, México; ICML-UNAM 3641, un espécimen, Rocas Consag, golfo de California, México, $31^{\circ} 07^{\prime} 00.12$ " N \& 114²9'59.87" W; ICML-UNAM 3643, un espécimen, punta Arbolada, golfo de California, México; ICMLUNAM 3932, un espécimen, bahía Petatlán, Guerrero, México, 17³4’00.12” N \& 101³0’00" W; ICML-UNAM 4469, cuatro especímenes, punta Arboleda, golfo de California, México; ICML-UNAM 5435, un espécimen, punta Mita, Nayarit, México.

Material tipo: Holotipo LACM 28.

Localidad tipo: Cabo San Francisco, Ecuador, Estación 850-38 (Deichmann, 1941).

Distribución geográfica: Cabo San Francisco, Ecuador (Deichmann, 1941).

Distribución batimétrica: $4.5 \mathrm{~m}$ (Deichmann, 1941).

Comentarios: Se amplía su límite de distribución batimétrica en su límite más profundo $(50 \mathrm{~m})$, y su distribución latitudinal a su límite más norteño (Rocas Consag, golfo de California).

Pseudocnus curatus (Cowles, 1907)

(Fig. 1E, 1F, 1G, 1H, Fig. 3)

Cucumaria curata Cowles, 1907: 8; Panning, 1949: 416; Lambert, 1995: 438; Bergen, 
1996: 21; Arndt, Marquez, Lambert, \& Smith, 1996: 434-435.

Pseudocnus curatus.- Lambert, 1997: 74-77; O’Loughlin, Mackenzie, Paulay, \& Vandenspiegel, 2014: 46; Mjobo, 2016: 21, Figs. 3-4.

Diagnosis (modificada de Mjobo, 2016): Cuerpo cilíndrico (Fig. 1E). Pies ambulacrales contraídos. Con 10 tentáculos, los dos ventrales son más pequeños (Fig. 1E). Presenta una vesícula de Poli. Las espículas de la pared del cuerpo son placas abollonadas, multiperforadas de la superficie dorsal y ventral, algunas de ellas con prolongaciones anteriores (Fig. 1F), también botones multiperforados (Fig. 1G). El introverso presenta barrotes perforados, algunos triradiados y barrotes curvos con perforaciones en las puntas (Fig. 1H).

Referencias de identificación: Panning, 1949: 416; Lambert, 1995: 438; Lambert, 1997: 74-77, Figs. 36, 37.

Material examinado: Cuatro especímenes. ICML-UNAM 132, un espécimen, Acapulco, Guerrero, México, 16 $6^{\circ} 51^{\prime} 26.38^{\prime \prime} \mathrm{N} \&$ 9953'3.80" W; ICML-UNAM 5.76.25, tres especímenes, El Almacén, Zihuatanejo, Guerrero, México, $17^{\circ} 38^{\prime} 00^{\prime \prime} \mathrm{N} \& 101^{\circ} 33^{\prime} 00^{\prime \prime} \mathrm{W}$.

Material tipo: Topotipo, MCZ HOL-178.

Localidad tipo: Cypress Point, Pacific Grove, California, Estados Unidos (Cowles, 1907).

Distribución geográfica: Desde el norte de Alaska, isla Dundas, islas Gnarled de Vancouver en la región de Victoria, Canadá; costa Sur de California, Monterey, Malpaso Creek, Estados Unidos (Lambert, 1997).

Distribución batimétrica: De 0 a $20 \mathrm{~m}$ (Lambert, 1997).

Comentarios: Se amplía su distribución latitudinal a su límite más sureño (Acapulco, Guerrero, México).

Pseudocnus dubiosus (Semper, 1868) (Fig. 2A, 2B, 2C, Fig. 3)

Cucumaria dubiosa Semper, 1868: 238; Deichmann, 1941: 81 .
Pseudocnus dubiosus dubiosa.- Panning, 1951: 73 .

Pseudocnus dubiosus dubiosus.- Panning, 1962: 59-60.

Pseudocnus dubiosus.- Pawson, 1964: 457-459; O'Loughlin et al., 2014: 46; Mjobo, 2016: 32, Figs. 8-10; Alvarado et al., 2017: 281.

Non Pseudocnus dubiosus Panning, 1949: 424.

Diagnosis (modificada de Pawson, 1964): Cuerpo cilíndrico (Fig. 2A). Con 10 tentáculos, los dos ventrales son más pequeños (Fig. 2A). Anillo calcáreo simple. Dos vesículas de Poli. Pies ambulacrales restringidos a las zonas ambulacrales. Las espículas de la zona dorsal son placas abollonadas con prolongaciones anteriores, en la parte ventral hay botones abollonados (Fig. 2B); introverso con barrotes perforados, curvados y en los tentáculos presenta barrotes perforados, irregulares, algunos trirradiados (Fig. 2C).

Referencias de identificación: Semper, 1868: 238, lám. 39, Fig. 19; Deichmann, 1941: 81, lám. 11, Figs. 1-2; Panning, 1951: 73-80, Figs. 1-3; Panning, 1962: 59-60, Figs. 1-2; Pawson, 1964: 20; Mjobo, 2016: 31, Figs. 8A, 8C, 9A, 9B, 10A, 10B.

Material examinado: 34 especímenes. ICML-UNAM 2103, 21 especímenes, lado Sur de la isla Pájaros, Mazatlán, Sinaloa, México; ICML-UNAM 2129, un espécimen, isla del Venado lado hacia isla Pájaros, Mazatlán, Sinaloa, México; ICML-UNAM 3122, un espécimen, en el cuello entre isla Venados y la isla Lobos, Mazatlán, Sinaloa, México; ICML-UNAM 3806, siete especímenes, al S.E. de la isla de Chivos a $80 \mathrm{~m}$ de la isla Chivos, Mazatlán, Sinaloa, México, 2311'6.13" N \& 106 $24^{\prime} 30.25^{\prime \prime} \mathrm{W}$; ICML-UNAM 3807, dos especímenes, parte este de isla Cardones, Mazatlán, Sinaloa, México, 2310'44.86" N \& 106²4'11.47' W; ICML-UNAM 1413, dos especímenes, Puerto Vallarta, Jalisco, México, $20^{\circ} 37^{\prime} 14.51^{\prime \prime} \mathrm{N} \& 105^{\circ} 14^{\prime} 2.24$ ” W.

Material tipo: Holotipo, se considera perdido (Samyn, Smirnov, \& Massin, 2013). Sintipos ZMH E6581 (Mjobo, 2016). 


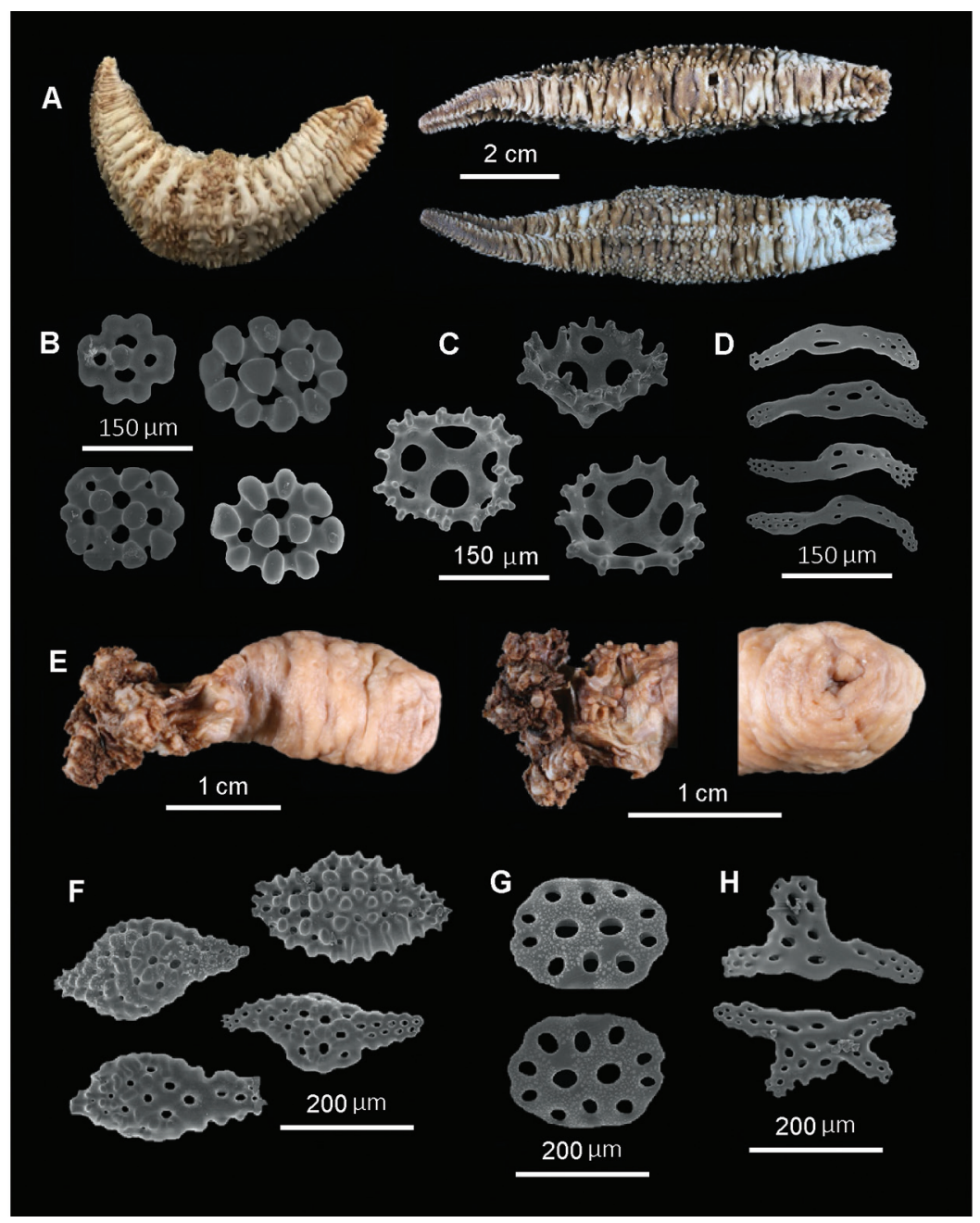

Fig. 1. A-D. Leptopentacta nina Deichmann, 1941 (ICML-UNAM 2379). A. Espécimen completo (vista lateral, dorsal y ventral). B. Botones lisos y abollonados de la superficie dorsal. C. Canastas de la superficie ventral. D. Barrotes perforados del introverso y tentáculos. E-H. Pseudocnus curatus (Cowles, 1907) (ICML-UNAM 132). E. Espécimen completo (vista dorsal, región anterior y posterior). F. Placas abollonadas de la superficie dorsal. G. Botones multiperforados de la superficie ventral. H. Barrotes perforados del introverso y tentáculos.

Fig. 1. A-D. Leptopentacta nina Deichmann, 1941 (ICML-UNAM 2379). A. Complete specimen (lateral, dorsal and ventral views). B. Knobbed and smooth buttons of dorsal surface. C. Baskets of the ventral surface. D. Perforated rods of the introvert and tentacles. E-H. Pseudocnus curatus (Cowles, 1907) (ICML-UNAM 132). E. Complete specimen (dorsal view, anterior and posterior region). F. Knobbed plates of the dorsal surface. G. Multi-perforated buttons of the ventral surface. H. Perforated rods of the introvert and tentacles.

Localidad tipo: Perú (Deichmann, 1941).

Distribución geográfica: Desde Costa Rica hasta Chile (Deichmann, 1941; Paredes, Tarazona, Canahuire, Romero, \& Cornejo, 1988; Solís-Marín et al., 2013).
Distribución batimétrica: De 0 a 300 m (Pawson, 1964; Solís-Marín et al., 2013).

Comentarios: Se amplía su rango de distribución latitudinal a su punto más norteño (Sinaloa, México). 
Pseudocnus lubricus (Clark, 1901)

(Fig. 2D, 2E, 2F, Fig. 3)

Cucumaria lubrica Clark, 1901: 334-335; Cherbonnier, 1951: 41-42.

Cucumaria fisheri astigmata Wells, 1924: 114.

Pseudocnus lubricus.- Lambert, 1997: 78; O’Loughlin et al., 2014: 45; Mjobo, 2016: 24-25 Figs. 5-7.
Diagnosis (modificada de Lambert, 1997): Cuerpo cilíndrico (Fig. 2D). Con 10 tentáculos, los dos ventrales son más pequeños (Fig. 2D). Anillo calcáreo simple, sin prolongaciones posteriores. Pies ambulacrales dispuestos en la zona ambulacral e interambulacral. Espículas de la pared del cuerpo con forma de placas abollonadas con una prolongación tipo cono (Fig. 2E). En el introverso las espículas presentan forma de barrotes irregulares trirradiados.

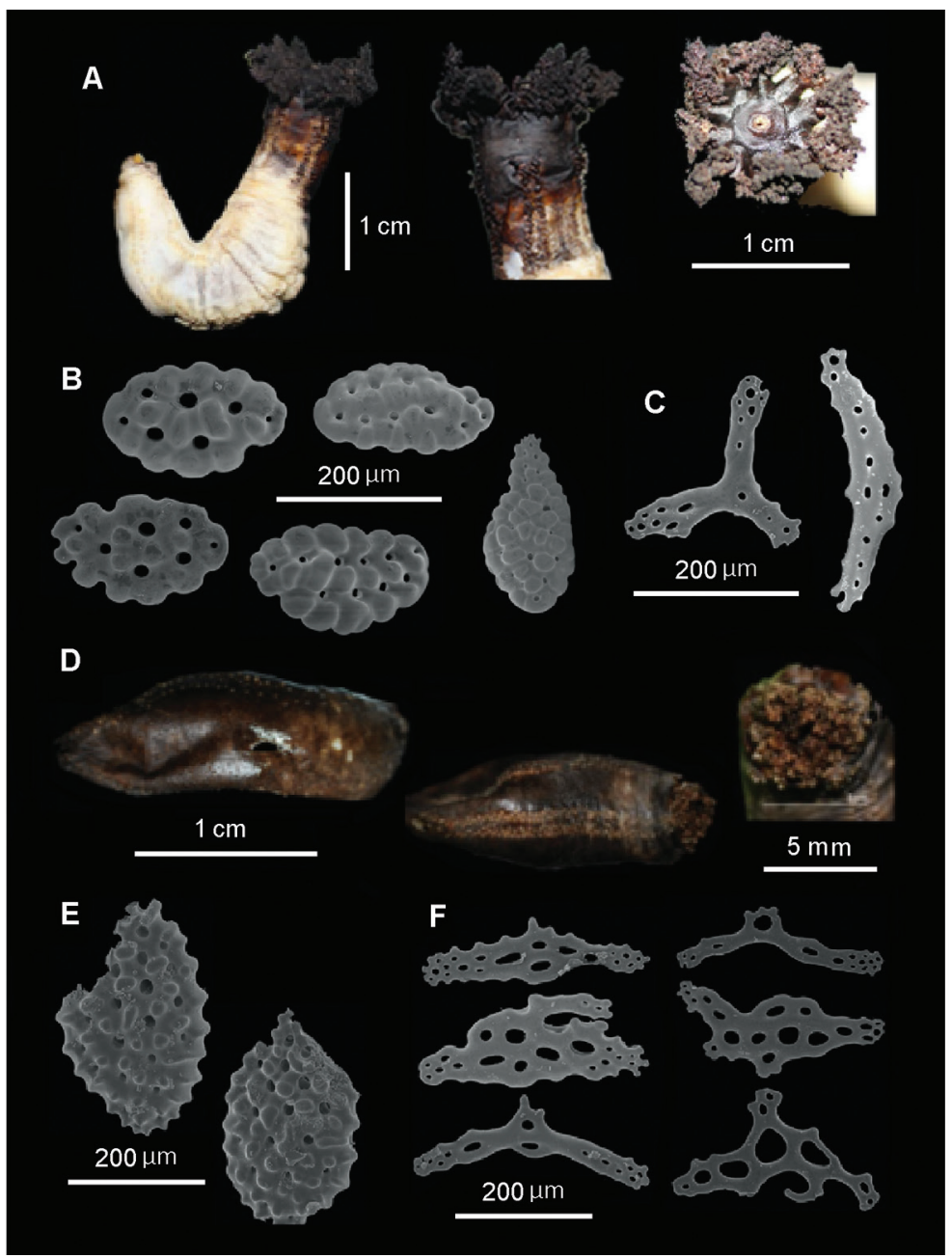

Fig. 2. A-C. Pseudocnus dubiosus (Semper, 1868) (ICML-UNAM 2103). A. Espécimen completo (vista lateral, introverso y corona de tentáculos). B. Placas abollonadas de la superficie dorsal y ventral. C. Barrotes perforados del introverso y tentáculos. D-F. Pseudocnus lubricus (Clark, 1901) (ICML-UNAM 4111). D. Espécimen completo (vista dorsal, ventral y tentáculos). E. Placas abollonadas de la superficie dorsal y ventral. F. Barrotes perforados del introverso y tentáculos.

Fig. 2. A-C. Pseudocnus dubiosus (Semper, 1868) (ICML-UNAM 2103). A. Complete specimen (lateral, introvert and tentacles). B. Knobbed plates on the dorsal and ventral surface. C. Perforated bars of the introvert and tentacles. D-F. Pseudocnus lubricus (Clark, 1901) (ICML-UNAM 4111). D. Complete specimen (dorsal, ventral view and tentacles). E. Knobbed plates of the dorsal and ventral surface. F. Perforated rods of the introvert and tentacles. 


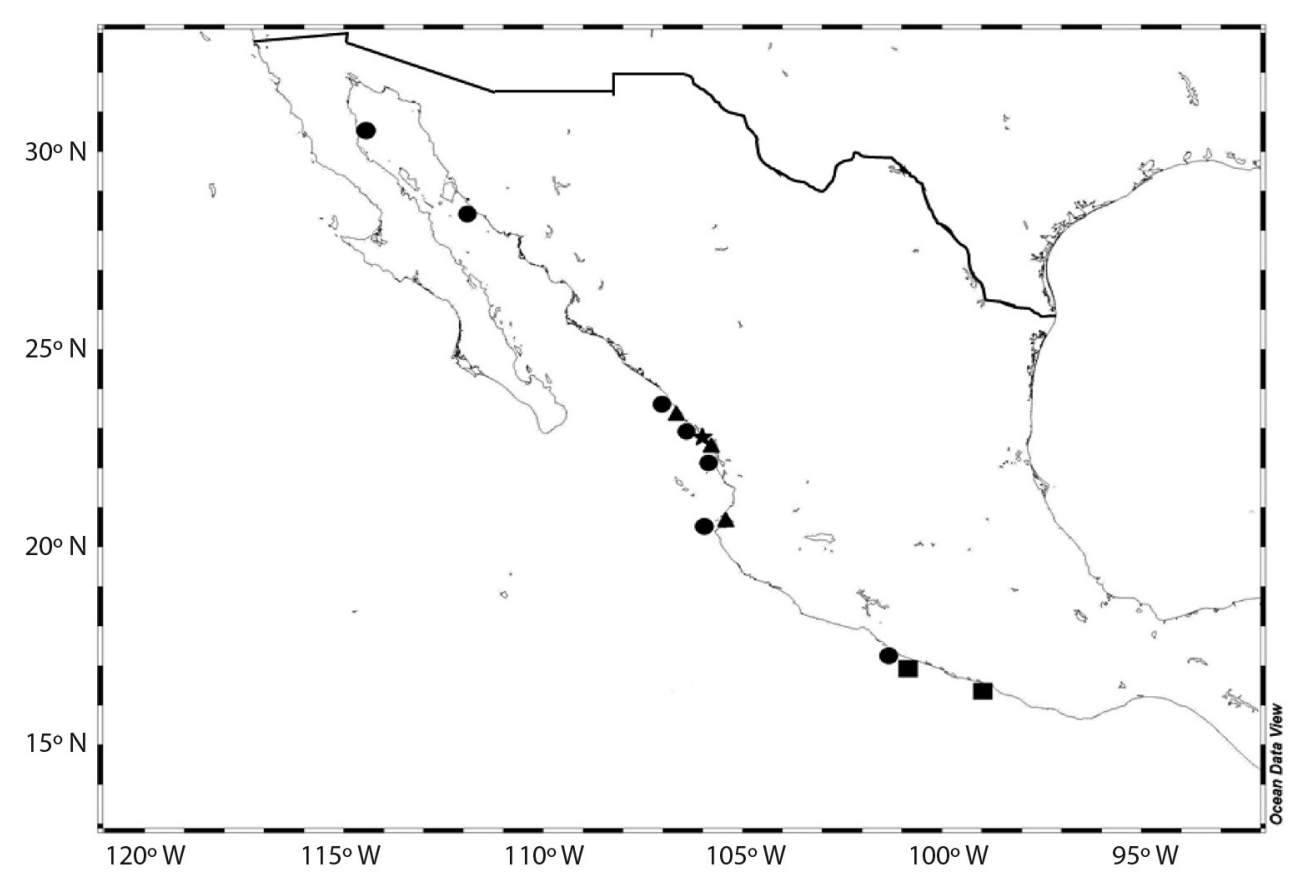

Fig. 3. Mapa del Pacífico mexicano mostrando los nuevos registros de las especies: Leptopentacta nina (círculo), Pseudocnus curatus (cuadrado), Pseudocnus dubiosus (triángulo) y Pseudocnus lubricus (estrella).

Fig. 3. Map of the Mexican Pacific showing the new records of the species: Leptopentacta nina (circle), Pseudocnus curatus (square), Pseudocnus dubiosus (triangle) and Pseudocnus lubricus (star).

Los tentáculos presentan barrotes perforados (Fig. 2F).

Referencia de identificación: Clark, 1901: 334, Lám. 4, Figs. 21-28; Cherbonnier, 1951: 41-43, Lám. 20, Figs. 1-9; Lambert, 1997: 78, Figs. 38, 39

Material examinado: ICML-UNAM 4111, cuatro especímenes, este de la isla Chivos, Mazatlán, Sinaloa, México, 2311'6.13" N \& $106^{\circ} 24^{\prime} 30.25^{\prime \prime} \mathrm{W}$.

Material tipo: Sintipos, MCZ HOL-202.

Localidad tipo: Pudget Sound, California, Estados Unidos (Clark, 1901).

Distribución geográfica: Desde Alaska, Columbia Británica, Canadá hasta California, Estados Unidos (Lambert, 1990, 1997).

Distribución batimétrica: De 0 a $78 \mathrm{~m}$ (Lambert, 1997).

Comentarios: Se amplía su distribución geográfica hasta su límite más sureño (Mazatlán, Sinaloa, México).

\section{DISCUSIÓN}

En trabajos previos (Honey-Escandón et al., 2008; Solís-Marín et al., 2009; SolísMarín et al., 2017) se habían reportado para el Pacífico mexicano nueve especies de la familia Cucumariidae. Uno de los caracteres diagnósticos de la familia Cucumariidae es la presencia de 10 tentáculos dendríticos (Ludwig, 1894), por lo anterior Smirnov (2012) transfirió a los géneros Abyssocucumis Heding, 1942 y Neocucumis Deichmann, 1944 a la familia Thyonidiidae por presentar de 15 a 25 tentáculos dendríticos, dando como resultado que las especies $A$. abyssorum y $N$. veleronis se excluyan de la familia Cucumariidae, dejando en siete el número de especies de cucumáridos reportadas para el Pacífico mexicano en trabajos previos.

Al momento de revisar los especímenes identificados bajo el nombre de T. peruana 
depositados en la Colección Nacional de Equinodermos, UNAM (ICML-UNAM 5.22.1; ICML-UNAM 2379, ICML-UNAM 3320, ICML-UNAM 3640, ICML-UNAM 3641, ICML-UNAM 3643, ICML-UNAM 3932, ICML-UNAM 4469 y ICML-UNAM 5435), se constató que la configuración espicular (botones lisos y abollonados ligeramente irregulares, canastas), así como la morfología externa e interna, correspondía más bien a $L$. nina (Fig. 1A, 1B, 1C, 1D), esta especie había sido reportada por Deichmann (1941) exclusivamente para aguas ecuatorianas, sin embargo, con los registros del presente trabajo, se amplía su distribución latitudinal más al norte (Rocas Consag, golfo de California, México), constituyendo este el primero registro de la especie para el Pacífico mexicano. Considerando que los registros de los especímenes identificados como $T$. peruana para México realizados por Caso (1986) y Solís-Marín et al. (2009) corresponden a ejemplares mal identificados, y si consideramos que son los únicos ejemplares que validan su distribución en dichas latitudes, se concluye que $T$. peruana no se distribuye dentro de los límites del Pacífico mexicano. Es probable que $L$. nina haya sido confundida con $T$. peruana por todos estos años dada su apariencia externa, ambas especies poseen los pies ambulacrales (no retráctiles) restringidos a las zonas ambulacrales, pero al revisar la configuración espicular de ambas se revela la verdadera identidad taxonómica.

Pseudocnus dubiosus fue descrita con ejemplares provenientes de Perú. Los caracteres principales que la diferencian del resto de sus congéneres es la presencia de placas abollonadas en la pared del cuerpo, las cuales son multiperforadas (Fig. 2B), con una prolongación posterior a veces presente, $\mathrm{y}$ por poseer barrotes triradiados en los tentáculos (Fig. 2C). El holotipo está perdido (Samyn et al., 2013), sin embargo, Mjobo (2016) presenta fotografías de microscopía electrónica de las espículas de la pared del cuerpo procedentes de los sintipos resguardados en el $\mathrm{ZMH}$, lo anterior nos ayudó a confirmar la identidad taxonómica de los ejemplares del Pacífico mexicano. La distribución geográfica de esta especie según Deichmann (1941) y Panning (1962) va desde aguas de centro y Sudamérica, hasta las islas Maldivas. Los ejemplares adjudicados a esta especie provenientes de las islas Maldivas por Deichmann (1941) y Lambert (2009) (como Pseudocnus dubiosus leoninus (Semper, 1868)), probablemente correspondan a Pentactella leonina (Semper, 1868), ya que esta última posee placas abollonadas con prolongaciones anteriores parecidas a las presentes en $P$. dubiosus lo que quizá confundió a los autores. La presencia de P. dubiosus en Perú y Costa Rica se puede constatar por los trabajos publicados (e.g. Prieto-Rios, 2010). De acuerdo con los especímenes revisados en este trabajo, se amplía la distribución latitudinal de esta especie a su límite más norteño (Mazatlán, Sinaloa, México).

Como se puede constatar en lo antes expuesto, han existido muchos problemas para identificar a las especies de la familia Cucumariidae a través del tiempo y en áreas de Pacífico Americano, es por ello que a continuación se mencionan los principales caracteres taxonómicos para identificar correctamente a una especie de esta familia: presencia de 10 tentáculos dendríticos; anillo calcáreo simple; presencia de botones abollonados en la pared del cuerpo, y algunas especies presentan también canastas; los tentáculos e introverso presentan barrotes perforados (algunos triradiados).

El Pacífico mexicano cuenta actualmente con 12 especies de la familia Cucumariidae. Este grupo taxonómico ha sido generalmente poco estudiado en todo el mundo, quizá lo anterior se deba a sus hábitos y comportamientos crípticos. En la actualidad, los límites morfológicos entre especies no se encuentran bien definidos. La morfología externa de los organismos, al momento de recolectarlos, enmascara la diversidad del grupo.

El listado de cucumáridos de México aún no se concluye, existen zonas marinas aún no exploradas (someras y profundas). La importancia de este trabajo radica en dar conocer la diversidad de la familia Cucumariidae del Pacífico mexicano. 
Declaración de ética: los autores declaran que todos están de acuerdo con esta publicación y que han hecho aportes que justifican su autoría; que no hay conflicto de interés de ningún tipo; y que han cumplido con todos los requisitos y procedimientos éticos y legales pertinentes. Todas las fuentes de financiamiento se detallan plena y claramente en la sección de agradecimientos. El respectivo documento legal firmado se encuentra en los archivos de la revista.

\section{AGRADECIMIENTOS}

A Ana Isabel Bieler Antolín (Laboratorio de Microcine de la Facultad de Ciencias, UNAM) por el apoyo brindado en la toma de fotografías de los ejemplares de pepinos de mar. A Berenit Mendoza Garfias (Laboratorio de Microscopía Electrónica en el Instituto de Biología, UNAM) por su apoyo en la toma de fotografías de Microscopía Electrónica de Barrido. A Alicia Durán González y Ma. Esther Diupotex Chong, técnicos académicos de la Colección Nacional de Equinodermos "Dra. María Elena Caso Muñoz" ICML, UNAM, por el apoyo brindado para la realización de este proyecto. A Penélope Ayala-Aguilera por el apoyo brindado para la realización del mapa. CACV (becario 666781) agradece al Consejo Nacional de Ciencia y Tecnología (CONACyT) por la beca de doctorado 722925 .

\section{RESUMEN}

\section{Nuevos registros de la familia Cucumariidae (Holothuroidea: Dendrochirotida) para el Pacífico mexicano}

Introducción: Los cucumáridos son un grupo cosmopolita de pepinos de mar que habitan en sedimentos rocosos-arenosos, desde la zona intermareal hasta la zona abisal. Objetivo: Presentar nuevos registros para el Pacífico mexicano. Métodos: Se utilizó estereoscopio y microscopía electrónica de barrido para mostrar la morfología externa e interna de especímenes de la colección de la Universidad Nacional Autónoma de México. Resultados: Presentamos una sinopsis taxonómica de cucumáridos en el Pacífico mexicano y revisamos su taxonomía en las espículas. Se confirma la identidad taxonómica de Pseudocnus curatus, Pseudocnus dubiosus y Pseudocnus lubricus y se descarta la presencia de Trachythyone peruana. Conclusiones: Se presentan cuatro nuevos registros para el Pacífico mexicano: Leptopentacta nina, Pseudocnus curatus, Pseudocnus dubiosus y Pseudocnus lubricus.

Palabras clave: pepinos de mar; Pacífico mexicano; biodiversidad; taxonomía; morfología.

\section{REFERENCIAS}

Alvarado, J.J., Solís-Marín, F.A., \& Ahearn, C. (2010). Echinoderm (Echinodermata) diversity in the Pacific coast of Central America. Marine Biodiversity, 40(1), 45-56.

Alvarado, J.J., Chacón, L., Solís-Marín, F.A., PinedaEnríquez, T., Caballero-Ochoa, A.A., Solano-Rivera, S., \& Romero-Chaves, R. (2017). Equinodermos del Museo de Zoología de la Universidad de Costa Rica. Revista Biología Tropical, 65(1), 272-287.

Arndt, A., Marquez, C., Lambert, P., \& Smith, M.J. (1996). Molecular phylogeny of Eastern Pacific sea cucumbers (Echinodermata: Holothuroidea) based on mitochondrial DNA sequence. Molecular Phylogenetics and Evolution, 6(3), 425-437.

Bergen, M. (1996). Class Holothuroidea. Taxonomic Atlas of the Benthic Fauna of the Santa Maria Basin and the Western Santa Barbara Channel, 14, 195-250.

Borrero-Pérez, G.H., Benavides-Serrato, M., \& DíazSánchez, C.M. (2012). Equinodermos del Caribe colombiano II: Echinoidea y Holothuroidea. Serie de Publicaciones Especiales de Invemar, 30, 250.

Caso, M.E. (1961). Los equinodermos de México (Tesis doctoral). Facultad de Ciencias, Universidad Nacional Autónoma de México, México.

Caso, M.E. (1984). Descripción de un género nuevo y una nueva especie de holoturoideo. Parathyonacta gen. nov. y Parathyonacta bonifaznuñoi sp. nov.; colectada en la campaña oceanográfica SIPCO III a bordo del Buque Oceanográfico "El Puma". Anales del Centro de Ciencias del Mar y Limnología, Universidad Nacional Autónoma de México, 11(1), 181-210.

Caso, M.E. (1986). Los equinodermos del Golfo de California colectados en las campañas SIPCO I-II-III a bordo del B/O "El Puma". Anales del Instituto de Ciencias del Mar y Limnología. Universidad Nacional Autónoma México, 13(1), 91-184.

Cherbonnier, G. (1951). Les Holothuries de Lesson. 1er Note. Bulletin du Muséum d'Histoire Naturelle, 23(3), 295-301.

Clark, H.L. (1901). Echinoderms from Puget Sound: Observations made on the Echinoderms collected by the parties from Columbia University, in Puget Sound 
in 1896 and 1897. Proceedings of the Boston Society of Natural History, 29(15), 323-337.

Cowles, R.P. (1907). Cucumaria curata sp. nov. Johns Hopkins University Circular, 195, 8-9.

De Blainville, H.M.D. (1830). Zoophytes. Dictionnaire des Sciences naturelles, dans lequel on traiteméthodiquement des différentsêetres de la nature, 60, 1-546.

Deichmann, E. (1941). The Holothuroidea collected by the Velero III during the years 1932 to 1938. Part I. Dendrochirota. Allan Hancock Pacific Expeditions, 8(3), 61-195.

Deichmann, E. (1944). Urodemas bifurcatum, a new holothurian from South Africa, with a revision of the genus Urodemas Selenka. Annals Magazine Natural History 11th Series, 11, 731-737.

Heding, S.G. (1942). Holothurioidea. Part II. Aspidochirota - Elasipoda - Dendrochirota. The Danish Ingolf expedition 1895-1896. Hagerup, Copenhagen, Denmark, 4(13), 3-39.

Heding, S.G. \& Panning, A. (1954) Phyllophoridae. Eine Bearbeitung der polytentaculaten dendrochiroten Holothurien des zoologischen Museums in Kopenhagen. Spolia Zoologica Musei Hauniensis, 13, 1-209.

Honey-Escandón, M., Solís-Marín, F.A., \& LaguardaFigueras, A. (2008). Equinodermos (Echinodermata) del Pacífico Mexicano. Revista de Biología Tropical, 56(3), 57-73.

Lambert, P. (1990). A new combination and synonymy for two subspecies of Cucumaria fisheri Wells (Echinodermata: Holothuroidea). Proceedings of the Biological Society of Washington, 103(4), 913-921.

Lambert, P. (1995). Collections, Research Paper and Special Projects. Sea cucumbers. Taxing Problems. Curator. Invertebrate Zoology. Vancouver: The Royal British Columbia Museum.

Lambert, P. (1997). Handbook of Sea Cucumbers of British Columbia, Southeast Alaska and Puget Sound. Vancouver: Royal British Columbia Museum UBC Press.

Lambert, P. (2009). Holothuroidea. En V. Häussermann (Ed.), Marine benthic fauna of Chilean Patagonia: Illustrated identification guide (pp. 860-880). Santiago, Chile: Nature in Focus.

Ludwig, H. (1894). The Holothurioidea. Reports on an Exploration off the West Coast of Mexico, Central and South America, and off the Galapagos Islands, in Charge of Alexander Agassiz, by the U. S. Fish Commission Steamer "Albatross", during 1891. XII. Memoirs of the Museum of Comparative Zoology, 17(3), 1-183.

Miller, A.K., Kerr, A.M., Paulay, G., Reich, M., Wilson, N.G., Carvajal, J.I., \& Rouse, G.W. (2017).
Molecular phylogeny of extant Holothuroidea (Echinodermata). Molecular Phylogenetics and Evolution, $111,110-131$.

Mjobo, S. (2016). A re-appraisal of the holothuroid genera Pseudocnus Panning, 1949 and Pseudocnella Thandar, 1987 based on morphological and for the latter, also molecular evidence (Echinodermata: Holothuroidea: Dendrochirotida: Cucumariidae) (Tesis de Maestría). School of Life Sciences, College of Agriculture, Engineering and Science, University of KwaZulu-Natal, Durban Republica de Sudáfrica.

Nava, H., Solís-Marín, F.A., Fuentes-Farias, A.L., CruzBarraza, J.A., Bastida-Zavala, J.R., LaguardaFigueras, A., ... Conejeros-Vargas, C.A. (2019). Invertebrados marinos. En A. Cruz-Angón, K.A. Nájera-Cordero \& E.D. Melgarejo (Coord.), La Biodiversidad en Michoacán. Estudio de Estado 2, vol. II (pp. 291-306). México: CONABIO.

O’Loughlin, P.M., Mackenzie, M., Paulay, G., \& Vandenspiegel, D. (2014). Four new species and a new genus of Antarctic sea cucumbers with taxonomic reviews of Cladodactyla, Pseudocnus, Paracucumidae and Parathyonidium (Echinodermata: Holothuroidea: Dendrochirotida). Memoirs of Museum Victoria, 72, 31-61.

Panning, A. (1949). Versuch einer Neuordnung der Familie Cucumariidae (Holothuroidea, Dendrochirota). Zoologische Jahrbücher Abteilung für Systematik, ökologie und Geographie der Tiere, 78(4), 404-470.

Panning, A. (1951). Über Pseudocnus leoninus (Semper) und verwandte Arten. Zoologischer Anzeiger Bd, 146(3-4), 73-80.

Panning, A. (1962). Bermerkungen über die HolothurienFamilie Cucumariidae (Ordnung Dendrochirota) 3 Teil. Die Gattung Pseudocnus (Panning 1949). Mitteilungen aus dem Hamburgischen Zoologischen Museum und Institut, 60, 57-80.

Paredes, C., Tarazona, J., Canahuire, E., Romero, L., \& Cornejo, O. (1988). Invertebrados macrobénticos del área de Pisco, Perú. En H. Salzwedel \& A. Landa (Eds.), Recursos y dinámica del ecosistema de afloramiento peruano (pp. 121-132). Perú: Boletín Extraordinario, Instituto del Mar del Perú.

Pawson, D.L. (1964). The Holothuroidea collected by the Royal Society Expedition to Southern Chile, 19581959. Pacific Science, 18(4), 453-470.

Pawson, D.L. (1982). Holothuroidea. En S.P. Parker (Ed.), Synopsis and classification of living organisms (pp. 813-818). Nueva York, E.U.A.: McGraw-Hill.

Pawson D.L., \& Fell, H.B. (1965). A revised classification of the Dendrochirote holothurians. Breviora, 214, $1-7$. 
Prieto-Rios, E. (2010). Taxonomía de Holothuroidea (Echinodermata) del mar del Perú (Tesis profesional). Universidad Nacional Mayor de San Marcos, Lima, Perú.

Samyn, Y., Smirnov, A., \& Massin, C. (2013). Carl Gottfried Semper (1832-1893) and the location of his type specimens of sea cucumbers. Archives of Natural History, 40(2), 324-339.

Semper, C. (1868). Holothurien. Reisen im Archipel der Philippinen. Zweiter Theil. Wissenschaftliche Resultate. Erster Band. Leipzig: W. Engelmann.

Smirnov, A.V. (2012). System of the Class Holothuroidea. Paleontological Journal, 46(8), 793-832.

Solís-Marín, F.A., \& Laguarda-Figueras, A. (1999). Cucumaria flamma, a new species of sea cucumber from the central eastern Pacific (Echinodermata: Holothuroidea). Proceedings of the Biological Society of Washington, 112(4), 778-786.

Solís-Marín, F.A., Arriaga-Ochoa J.A., Laguarda-Figueras, A., Frontana-Uribe, S.C., \& Durán-González, A. (2009). Holoturoideos (Echinodermata: Holothuroidea) del Golfo de California. México: Instituto de Ciencias del Mar y Limnología, Universidad Nacional Autónoma de México y Comisión Nacional para el Conocimiento y Uso de la Biodiversidad.
Solís-Marín, F.A., Caballero-Ochoa, A.A., Frontana-Uribe, S.C., Laguarda-Figueras, A., \& A. Durán-González. (2017). Catálogo de autoridades taxonómicas de los equinodermos de México. México: Instituto de Ciencias del Mar y Limnología, Universidad Nacional Autónoma de México. Informe final, SNIBCONABIO, proyecto No. Z002.

Solís-Marín, F.A., Alvarado, J.J., Abreu-Pérez, M., Aguilera, O., Alió, J., Bacallado-Aránega, J.J., ... Williams, S.M. (2013). Appendix: A.1. Taxonomic list of the Echinoderms of the Pacific coast of Latin America. En J.J. Alvarado \& F.A. Solís-Marín (Ed.), Echinoderm Research and Diversity in Latin America (pp. 544-601). Berlin, Heidelberg: Springer.

Stimpson, W. (1864). Descriptions of new species of marine invertebrata from Puget Sound, collected by the naturalists of the North-West Boundary Commission, A.H. Campbell, Esq., Commissioner. Proceedings of the Academy of Natural Sciences of Philadelphia, 16, 153-161.

Théel, H. (1886). Report on the Holothurioidea collected by H.M.S. Challenger during the Years 1873-76. Part II. Report on the Scientific Results of the Voyage of H.M.S. Challenger during the years 1873-76. Zoology, 14(39), 1-290.

Wells, H. (1924). New species of Cucumaria from Monterey Bay, California. Annals and Magazine of Natural History, 14(79), 113-121. 